\title{
USING SMART CONTRACTS IN SMART ENERGY GRID APPLICATIONS
}

\author{
Panagiotis Giannakaris, \\ Panagiotis Trakadas*, \\ Theodore Zahariadis, \\ Panagiotis Gkonis, \\ Konstantinos Papadopoulos
}

Technological Educational Institute of Sterea Ellada,

Department of Electrical Engineering, Chalkis, Greece
Correspondence:

Panagiotis Trakadas

e-mail:

ptrakadas@teiste.gr

\begin{abstract}
:
The evolution of the energy production and distribution towards innovative decentralized models, dictates the introduction of emerging technologies to transform the conventional energy sector into smart. In this paper, blockchain technology is introduced, in the concept of utilization and associated benefits in smart grid sector. Moreover, potential applications of smart contracts in the smart metering environment are discussed as well. Blockchain-based technology including smart contracts, is an ideal candidate for matching energy demand and production at smart grid level. As such, consumers will have the ability to become prosumers with a significant role in the continuous smart grid stabilization, since in a smart metering environment, all the contracting nodes will be able to streamline their energy consumption according to the grid demand. In addition to that, by the introduction of smart contracts within the smart grid landscape, smart meters can go one step further, allowing customers to pay as they go, rather than relying on monthly assessments.
\end{abstract}

Keywords:

blockchain; smart contract; smart energy grid.

\section{INTRODUCTION}

During the last decade, a revolution has started that transformed the way that demand and supply of electricity is organized and offered to the prosumers. The large-scale transition towards renewable and decentralized energy brought a plethora of unforeseen changes in the energy sector. In order to cope with the associated challenges, energy industry is actively examining new business models and technological mechanisms for delivering new and better services to customers. Likewise, customers are seeking new ways to purchase energy at the lowest price and are willing to consume energy, generated from renewable sources. As such, relevant stakeholders are increasingly promoting new peer-to-peer trading mechanisms based on decentralized technologies. Blockchain is the most innovative, promising and popular technology to address these issues. It can be considered as an IT platform connecting energy producers and consumers in a decentralized manner, the so-called prosumers, that not only consume but also produce energy from several sources, such as rooftop photovoltaic panels, that can be inserted in the energy grid 
and consumed from other neighbors. In this decentralized concept, blockchain technology has the potential to widen the range of opportunities for market entrants to expand their businesses into the energy market [1].

This paper is the first step towards the realization of an M.Sc. thesis related to the introduction of smart contracts within the smart grid ecosystem and provides an overview of the solutions that blockchain-based technologies can offer to this new era of smart grid.

The rest of the paper is organized as follows: In section II, the main principles of blockchain technology are provided. In section III, the concept of smart contracts as well as the way that can be customized and utilized in the blockchain technology is described. Section IV analyzes the use of the smart contracts in a smart metering environment, while the benefits and drawbacks of the blockchain technology in the energy sector are clarified. Finally, section $\mathrm{V}$ concludes this position paper and explains future work.

\section{PRINCIPLES OF BLOCKCHAIN TECHNOLOGY}

While blockchain technology was originally devoted to digital currency applications, the research community soon realized that its usability can be advantageous to many societal, market and business sectors, including energy sector [2], [3], [4]. By storing blocks of information that are identical across the nodes that comprise a network, the blockchain is not be controlled by any single entity, but rather depends on the majority of the nodes participating in the network. Due to this decentralized approach, a blockchain-based network has no single point of failure.

Companies, worldwide, have started to explore the ways that blockchain technology could be used to provide new services to the market and enhance the existing ones. As it becomes apparent, blockchain technology is an ideal candidate for decentralized processes that require large networks and trust relationships between all parties, as the case of smart grid sector.

One of the main components of a smart grid is smart meters. Smart meters are the communication devices used as gateways between households and the electricity grid. Smart meters allow for the exchange of information both in terms of electricity consumption flows as well as information flows with respect to billing, prices and other related information. Moreover, in the current realization of a smart grid, a centralized marketplace for monitoring and balancing the supply and demand is needed. Instead of this centralized approach, smart contracts can be used to balance demand and supply in a decentralized approach without the need for a trusted third party.

In principle, a blockchain network consists of a number of computing nodes, where each node is using a client to validate and relay transactions between the users of the network, and store the latest copy of the digital ledger that is created from the validated transactions among the users of the blockchain. Fig. 1, depicts nodes comprising a simple blockchain network.

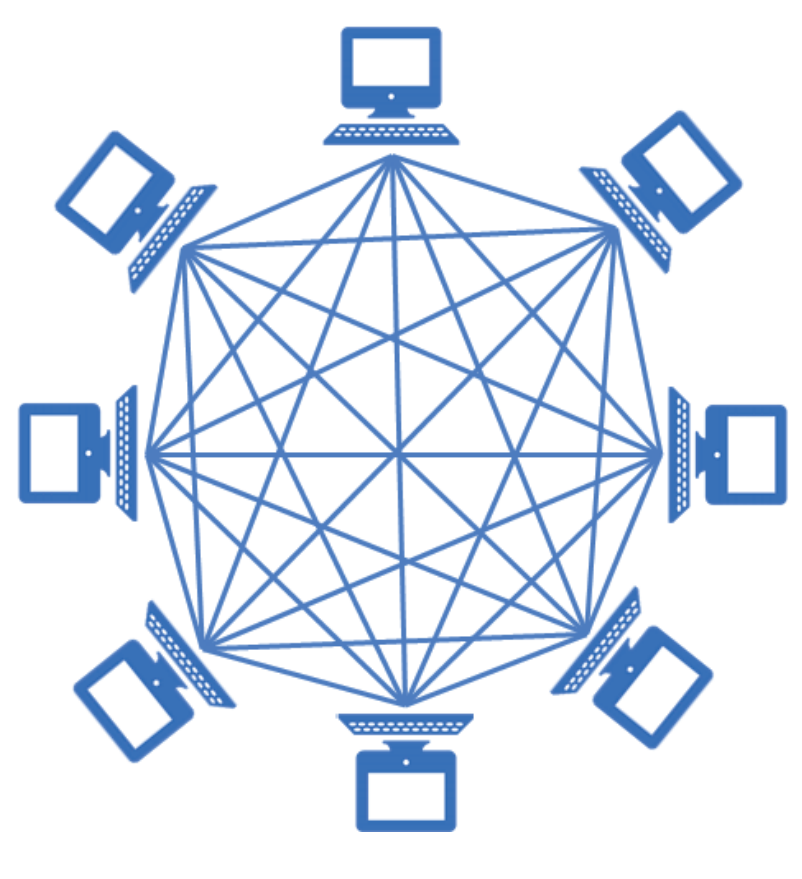

Figure 1. A blockchain-based network consisting of several nodes

The content of each block of the ledger is an aggregated set of data or, equivalently, an append-only list of records. Data are collected and processed to fit in a block, through a process called mining and each block could be identified using a cryptographic hash (digital fingerprint). The formed block will contain newly inserted information of the transaction, along with a hash of the previous block. Thus, the blocks can form a chain from the first block (Genesis Block) to the last one. In this way, all the data could be connected via a linked list structure. Fig. 2, depicts how hash of the blocks within a blockchain is calculated. 


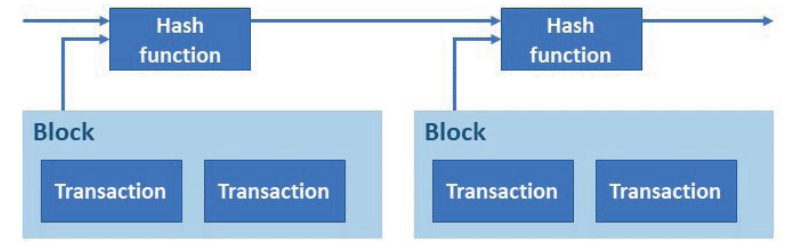

Figure 2. Illustration of blockchain hashing function

In the case of the Bitcoin blockchain, the block contains a header and relevant transaction data. A hash tree (or Merkle tree) of transactions is created and the hash of the root is included in the header. A hash tree is a full binary tree of a hash values. At the bottom level of the tree, each transaction has a node containing its hash value. After that, the tree is constructed in a way such that the parent node has a value of the hash of the data contained in its children concatenating together. In this way, a hash tree allows efficient and secure validation by constructing a Merkle tree path from the bottom level of the tree up to the root node. Fig. 3, illustrates a hash tree. In a blockchain, the block header has a field for previous block hash. Hence, each block will contain a reference of its previous block, and this could build up a chain of blocks. As it becomes obvious from its inception, a blockchain is resistant to modification of the data.

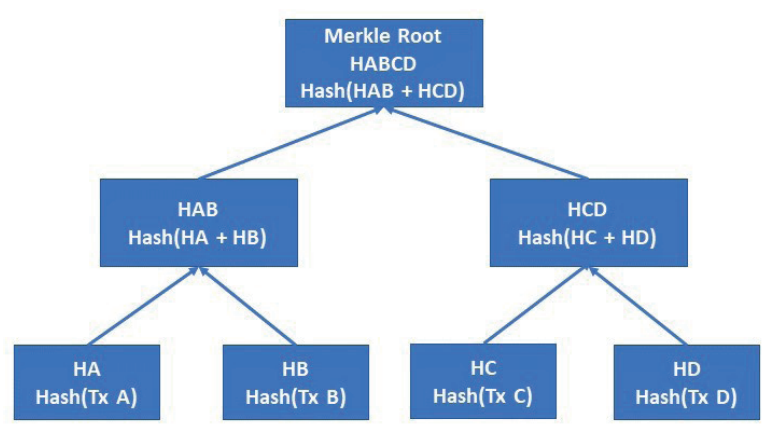

Figure 3. Illustration of a Hash Merkle tree

A blockchain is a type of a distributed ledger. The common characteristic between blockchain and distributed ledger technology is that the blocks are not maintained by any central authority. However, important differences may exist among the blockchain and distributed ledger technologies. In concrete terms, a distributed ledger is a database spread across all nodes of the network, where each node processes and stores an identical copy of the latest version of the ledger. Any updates to the ledger are performed independently and recorded by every node in the network. Afterwards, the nodes execute a voting process on these updates to ensure that the majority agrees with the conclusion reached. This voting and agreement methodology on the ledger is called consensus and is conducted by a consensus algorithm. Once consensus is reached, the distributed ledger updates itself and the latest, majority-agreed version of the ledger is saved on each node separately [5].

Given the former analysis, blockchain is one form of distributed ledger technology, but its structure differentiates it from other types of distributed ledgers. Data on a blockchain are grouped together and organized in blocks. The blocks are then linked to one another and secured using cryptographic algorithms. The structure of a blockchain allows data only to be appended to the database. Altering or deleting previously entered data on earlier blocks is impossible. Thus, given its unique characteristics, blockchain technology is well-suited for recording events, managing records, processing transactions, tracing assets, and voting without the need for the existence of a trusted third party.

Therefore, every blockchain is a distributed ledger, but not every distributed ledger is a blockchain. Each of these concepts requires decentralization and consensus among nodes. However, the blockchain organizes data in blocks, and updates the entries using an append-only structure.

\section{PRINCIPLES OF SMART CONTRACTS}

A few years after the introduction of blockchain concept, it became apparent that a decentralized ledger could be used for smart contracts (otherwise called self-executing contracts, or digital contracts). In this concept, contracts are independent pieces of software code (scripts) that are stored and replicated on the system and supervised by the network of computer nodes that comprise the blockchain. This mechanism is able to automatically activate terms of the contract (e.g. transferring money if and only if specific conditions and met). Thus, the exchange of any product or service can be facilitated by the use of smart contracts, in a transparent, unconflicted and immutable way while avoiding the inclusion of a trusted third party. In other words, smart contract is a computer code that runs on top of a distributed ledger and includes rules that the involved parties have agreed upon in order to interact with each other. In the case that these rules are satisfied, the smart contract agreement is automatically 
activated. In this way, the smart contract code verifies and executes the terms of the smart contract, providing a simple form of decentralized automation. More specifically, a smart contract can be considered as a mechanism that involves digital assets of the users that participate in the smart contract and these assets are exchanged among the participants when the rules of the smart contract are satisfied in a way that it is declaratively described within the smart contract code. In brief, the smart contracts have the ability to turn legal obligations into automated processes, guarantee a greater degree of security, reduce reliance on trusted intermediaries and lower the transaction costs.

In particular, energy industries provide real-world use cases to demonstrate the value of blockchain-based smart contract technology. In the energy industry, blockchain-based smart contracts can enable smart grids, microgrids, and other types of innovative grid management technologies by both providing the mechanism for automating value transmission and the means to streamline transaction administration.

\section{BLOCKCHAIN-BASED SMART CONTRACTS IN SMART GRID}

Energy industries provide real-world use cases to demonstrate the value of blockchain-based smart contract technology. In the energy industry, blockchainbased smart contracts can enable smart grids, microgrids, and other types of innovative grid management technologies by both providing the mechanism for automating value transmission and the means to streamline transaction administration.

Blockchain-based smart contracts, support the "as you go" pay smart meter concept. However, even in this case, certain vulnerabilities exist. For example, the smart meters' security is insufficient, due to their probably outdated protocols or the inability of the costumers to visit a payment machine in order to reload their payment cards. By contrast, smart contracts can augment the security of smart meters on a blockchain-based system, as well as to enhance the process of payment in a smart metering environment [6], [7]. Blockchain-based smart contracts, makes it possible for customers to arrange their payments on their phones, tablets or PCs, using blockchain-based smart contracts that execute when their remaining electrical power is reduced below a certain amount. In the near future, blockchain-based smart contracts could also be structured to be executed according to weather conditions, adjusting accordingly the credit amount of a specified consumer.
With a blockchain-based system, consumers would have direct access to an immutable record of their consumed energy, which could be compared with their historical usage. Therefore, consumers would be able to make the appropriate adjustments in specific parameters of the smart contracts, according to the current energy demands in a range of a few days. The immediate feedback of the energy usage at costumers, gives them the benefit to manage their energy assets with high accuracy.

In this approach, a blockchain based distributed ledger stores in a tamper proof manner the collected data of the energy consumption from smart metering devices, while self-enforcing smart contracts programmatically define the expected energy flexibility at the level of each prosumer. Blockchain demand side management is able to match demand and production at smart grid level.

In the case of the demand-response model of a smart grid, a smart contract can be observed as a piece of code that defines the appropriate actions of each producer in order to participate in such demand-response programs, accompanied by the rules for assuring the grid level balance between energy demand and energy production. These contracts are registered in the blockchain and are triggered by new energy transactions, which make each blockchain node to update its state, based on the results obtained after executing the smart contract. The involvement of each entity in a demand-response event takes place by the use of self-enforcing smart contracts. Such contracts, are defined and used to implement in a programmatic manner the levels of energy demand flexibility where energy providers may provide during demand-response events, associated with incentive and/ or penalty rates, as well as rules for balancing the energy demand with the energy production. The self-enforcing smart contracts are defined in a distributed fashion at the level of each energy provider voluntarily enrolled with the demand-response event and specify the expected energy demand and adjustments to its energy baseline. For each value provided and stored to a specific block by the smart metering device associated with the energy provider, the corresponding smart contract evaluates, by estimating the difference between the expected energy curve and actual monitored energy values.

In the case of significant variations, the appropriate actions are taken to rebalance the energy demand with the energy production. Thus, smart contracts act as a decentralized control mechanism. Based on the registered deviations in the local grid, new demand-response events are defined in a decentralized manner, providing 
also the rates for penalizing the entities that violate the smart contract and for rewarding those that handle their energy production with flexibility.

Moreover, blockchain distributed consensus, is used for verification of consumption and financial settlement, using the information gathered from the smart meters. This information is aggregated in blockchain blocks, and afterwards it is permanently registered and further replicated across the distributed ledger. Since the data structures offered by blockchain are based on hash pointers, the resulting benefit is that the entire ledger is entered in a tamper proof environment, that can be modified only by re-computing the hashes for all the following blocks, which is infeasible. Thus, one of the most important operations within the blockchain approach is that the majority of the network nodes must agree on the contents of the ledger, which in this case reflects the energy state of the smart grid.

In conclusion, compared with a traditional energy consumption contract, the blockchain based management of the smart energy grids, provides many solutions to a set of already identified problems that currently exist in this domain. The adoption of the Blockchain concepts will transform the smart grid into a democratic community, that no longer relies on a central authority but can take any decision through smart contract rules enforced and verified by each node of the grid. Moreover, it will provide transparency with respect to pricing models.

\section{VULNERABILITIES IN SMART METERING ENVIRONMENT}

As a transparent and decentralized transaction technology, blockchain may be able to provide the security that is missing in the design of many smart meters today. But is blockchain ready to be used as a communication protocol in the electrical grid? As it is mentioned below, smart meters rollout, reveals several vulnerabilities which may occur during the deployment of the smart grid.

- Communication protocols between smart meters and home appliances, are possible to be exposed to attacks by hackers.

- In the case, where the utilities follow an adverse incentive system that focuses on cost-savings rather than security, they may lack the personnel to properly assess the risks and mitigate the attacks.
- Detaching smart meter operations from the accountability of the grid operator, as it happens in some European countries, including Germany and the United Kingdom, may augment the lack of security.

- If an attack occurs, it is still unclear who is liable for it.

\section{CONCLUSIONS}

Blockchain technology and its derivatives, such as smart contracts and distributed ledger technologies, in general, is a promising technology that provides several opportunities across the market sectors but in particular in the smart grid domain. Blockchain smart contracts in energy management, will bring resilience and flexibility in the sale and purchase of electricity with clear benefits for both the industrial stakeholders and the customers. In particular, customers will have in hand plenty of useful information with respect to their daily energy usage and related costs. They will also be capable of planning their energy consumption and predict their payment accordingly.

On the downside, there are still open issues prior to the wide acceptance and implementation of smart contracts in the energy market. Blockchain based technology in energy sector, is still in an initial stage worldwide and lacking a pan-European regulatory framework that would allow this technology to show its full potentials. The changeover from the traditional way of energy payment to an ecosystem including new trading mechanisms, is something that it will need time for familiarity, not so much from young age persons but from older ones. The familiarity with smart phones which has taken place a decade ago, can definitely help the well establishment of blockchain smart contract technology, since the transactions and settles which have to do with the energy management, will be mostly held through smart phones or tablets. Nevertheless, with the pass of time, blockchain technology in energy sector as every other new one, will become a piece of our daily life.

This paper is categorizing the issues related to the inclusion of the smart contracts in the smart grid application domain in order to provide innovative security solutions and traceability on energy consumption. 


\section{ACKNOWLEDGEMENT}

This work has been performed in the framework of the DEFENDER (ICT-740898) and NRG5 (ICT-762013) projects, funded by the European Commission through the Horizon 2020 and 5G-PPP programmes.

\section{REFERENCES}

[1] D. Puthal, N. Malik, S. P. Mohanty, E. Kougianos, G. Das, "Everything you wanted to know about the blockchain: Its promise, components, processes, and problems", IEEE Consumer Electronics Mag., vol. 7, no. 4, pp. 6-14, July 2018.

[2] J. Wu, N.K. Tran, "Application of Blockchain Technology in Sustainable Energy Systems: An Overview", Sustainability, vol. 10, no. 9, 2018, doi: 10.3390/su10093067.

[3] M.S. Ali, M. Vecchio, M. Pincheira, K. Dolui, F. Antonelli, M.H. Rehmani, "Applications of Blockchains in the Internet of Things: A Comprehensive Survey", IEEE Communications Surveys \& Tutorials, December 2018, doi: 10.1109/ COMST.2018.2886932
[4] A. Pieroni, N. Scarpato, L. Di Nunzio, F. Fallucchi, M. Raso, "Smarter City: Smart Energy Grid based on Blockchain Technology", International Journal on Advanced Science, Engineering and Information Technology, vol. 8, no. 1, 2018, pages: 298-306, doi:10.18517/ijaseit.8.1.4954.

[5] T.M. Fernandez-Carames, and P. Fraga-Lamas, "A Review on the Use of Blockchain for the Internet of Things", IEEE Access, vol. 6, pp. 32979 - 33001, 2018, doi: 10.1109/ACCESS.2018.2842685.

[6] Z. Li, J. Kang, R. Yu, D. Ye, Q. Deng, and Y. Zhang, "Consortium Blockchain for Secure Energy Trading in Industrial Internet of Things", IEEE Trans. on Ind. Informatics, vol. 14, no. 8, 2018, pp. 3690 - 3700, doi 10.1109/TII.2017.2786307.

[7] S-K Kim, J-H Huh, "A Study on the Improvement of Smart Grid Security Performance and Blockchain Smart Grid Perspective", Energies, vol. 11, no. 8, 2018, doi: 10.3390/en11081 